\title{
Search for intrinsic variable stars in three open clusters: NGC 1664, NGC 6811 and NGC 7209
}

\author{
P. Van Cauteren ${ }^{1}$, P. Lampens ${ }^{2}$, C.W. Robertson ${ }^{3}$ and A. Strigachev ${ }^{4}{ }^{2}$ \\ ${ }^{1}$ Beersel Hills Observatory, Beersel, Belgium \\ ${ }^{2}$ Royal Observatory of Belgium, 3 Ringlaan, 1180 Brussels, Belgium \\ ${ }^{3}$ SETEC Observatory, Goddard, Kansas, USA \\ ${ }^{4}$ Institute of Astronomy, Bulgarian Academy of Sciences, Sofia, Bulgaria
}

\begin{abstract}
We report on new time-series CCD observations for three poorly studied open clusters from the STACC list. The collected light curves in the $\mathrm{V}$ filter of a large number of stars per field have been examined in detail in search for shortperiodic variable stars. In particular we looked for new $\delta$ Scuti-type candidates. First results for the open clusters NGC 1664, NGC 6811 and NGC 7209 are presented.
\end{abstract}

\section{Why study open clusters ?}

The study of variable stars in open clusters presents various advantages for the understanding of pulsation physics:

- open clusters with variable members provide comparative asteroseismological tests of stellar structure and evolution based on the implications of a common origin and formation of their stellar members;

- open clusters are ideally suited for performing high-accuracy differential photometry as well as 'CCD ensemble photometry' based on a large sample of comparison stars in the field-of-view.

Such studies moreover require long baseline differential photometry (to detect multiple frequencies combined with small amplitudes) as well as complementary multi-color photometry or high-resolution spectroscopy (to determine the basic stellar parameters) if one wishes to take full profit of these observations 
(Freyhammer et al. 2001). We selected three poorly studied open clusters from the STACC list (Frandsen \& Arentoft 1998) in order to perform a CCD survey using small telescopes (up to $1.3 \mathrm{~m}$ ) with the goal to search for intrinsic variable stars. These open clusters are visible from the Northern hemisphere and are furthermore prime targets for the detection of $\delta$ Scuti-type candidate stars (Frandsen \& Arentoft 1998).

On the other side, the presence of close neighboring stars in the (semi)crowded fields of the central parts of open clusters may affect the quality of the results and this could imply the need for a different reduction technique (point-spread function fitting instead of the aperture photometry approach).

\section{General information about the observed clusters}

\subsection{NGC 1664}

Is an extended open cluster of the STACC list with a rich central region (Frandsen \& Arentoft 1998). A recent proper motion study was performed by Baumgardt et al. (2000). The cluster membership was also investigated by Missana \& Missana (1998). It is situated at a distance of about 1200 pc and has a log age of 8.47 (Mermilliod 1995).

\subsection{NGC 6811}

Is an open cluster of the STACC list with many candidates in the instability strip (Frandsen \& Arentoft 1998). A proper motion study was performed by Sanders (1971), yielding 97 probable members. Glushkova et al. (1999) collected radial velocities in the field and identified seven new members. From their photoelectric photometric data they obtained a mean distance of $1040 \mathrm{pc}$ and an age of 0.7 Gyr based on 88 identified cluster members (log age $=8.85$ ).

\subsection{NGC 7209}

Is an open cluster of the STACC list with a poorly populated color-magnitude diagram (Frandsen \& Arentoft 1998). Several studies of proper motions in the cluster field exist: for example, Platais (1991) found 148 possible cluster members down to mag 15 while recent proper motion studies were performed by Baumgardt et al. (2000) and by Dias et al. (2001) based on the TYCHO-2 catalogue. Peña \& Peniche (1994) proposed the existence of two clusters, NGC 7209 $a$ and $b$, with a different reddening, age and distance based on Strömgren photometry of 54 stars in the cluster direction. Vancevicius et al. (1997) obtained photoelectric photometry in the Vilnius system for 96 probable members but do not support that conclusion: they estimated the mean reddening, the metalicity 
and derived a median distance of $1026 \pm 30 \mathrm{pc}$ and an age of $0.45 \mathrm{Gyr}$ (log age $=8.65$ ).

\section{Observations and photometric reduction}

The logbook of all observations, the telescopes and CCD cameras used are listed in Table 1. Except for the observatory Hoher List (HL) where a selfbuilt camera was employed $(2 \mathrm{~K} \times 2 \mathrm{~K}$; HoLiCam of the Observatorium Hoher List, University of Bonn, Germany), the other cameras are from Photometrics ( $1 \mathrm{~K} \times 1 \mathrm{~K}$; Skinakas, Crete) or SBIG (various ST's), equipping instruments with different focal lengths. We typically made use of a field-of-view with a size of $26^{\prime} \times 28^{\prime}$ (e.g. BHO $\left.40 \mathrm{~cm}\right)$ or $34^{\prime} \times 23^{\prime}$ (e.g. BHO $\left.25 \mathrm{~cm}\right)$.

Table 1: Log of the cluster observations

\begin{tabular}{|c|c|c|c|c|c|c|}
\hline NGC & Obs/Tel & CCD & Start & End & $\mathrm{Nr} / \mathrm{hrs}$ & Filter \\
\hline \multirow[t]{3}{*}{1664} & BHO40 & ST10E & $27-10-03$ & $10-12-03$ & 25.8 & $B, V$ \\
\hline & $\mathrm{BHO} 25$ & ST7 & $08-12-03$ & $08-12-03$ & 8.9 & V \\
\hline & HL100 & HoLiCam & $17-12-03$ & $17-12-03$ & 5.5 & V \\
\hline \multirow[t]{2}{*}{6811} & BHO40 & ST10E & $22-03-03$ & $27-09-03$ & 31.8 & $B, V$ \\
\hline & NAO70 & ST8 & 03-05-03 & 03-05-03 & 3.3 & V \\
\hline \multirow[t]{4}{*}{7209} & $\mathrm{BHO} 40$ & ST10E & $29-07-02$ & $20-08-03$ & 25.9 & $B, V$ \\
\hline & $\mathrm{BHO} 25$ & ST7 & 01-08-03 & $10-08-03$ & 31.9 & V \\
\hline & SETEC & ST8i & $12-08-02$ & 05-09-02 & 42.6 & V \\
\hline & SKI130 & $\mathrm{CH} 360$ & $19-08-03$ & 04-10-03 & 9.2 & V \\
\hline
\end{tabular}

All the frames were treated following the classical basic reduction scheme: a median dark/bias was subtracted from each science frame and these were then divided by a median flat-field. Aperture photometry was subsequently performed using various packages. Consequently, we intentionally avoided the stars with close neighbors on the collected images. First, for all the frames obtained at BHO, HL, SETEC and Skinakas, the aperture photometric package of MIRA AP was applied. Differential magnitudes with respect to a pre-selected comparison star were produced. For the frames acquired during one night in

\footnotetext{
${ }^{0}$ The MIRA AP software is produced by Axiom Research Inc.
} 
2003 with the Schmidt 50/70 telescope of the National Astronomical Observatory of Bulgaria (Tsvetkov et al. 1987), we gathered the differential data using the MOMF code (Kjeldsen \& Frandsen 1992). In a second step, CCD ensemble photometry was applied in order to produce differential magnitudes with respect to a comparison magnitude using a(n) (inhomogeneous) set of comparison stars (Honeycutt 1992). The figures shown below are based on the latter data.

A $V$ filter following Bessel's (1995) specifications was used for the time-series photometry. In addition, some nights were dedicated to the acquisition of the B-magnitudes with the aim to produce updated color-magnitude diagrams in the near future.

\section{Results}

\subsection{NGC 1664}

\subsubsection{Analysis}

The observed field of NGC 1664 is illustrated by Fig. 1. We used the numbering of the (WE)BDA (Mermilliod 1995; cf. http://www.obswww.unige.ch/webda/) database for identification purposes. 9 stars received an internal numbering. 284 stars were included in the reduction scheme and examined for rapid light variations. The CCD data consist of more than 300 differential magnitudes in the mean for most targets, with actual numbers varying between 39 (a few) and 516 (many) depending on the position in the field.

\subsubsection{Discussion of individual variable stars}

We report the detection of two new short-periodic variable stars in the field: $\mathrm{V} 1$ and $\mathrm{V} 2$. Both are possible $\delta$ Scutivariable stars. A period analysis of the $\mathrm{V}$-band data for star $\# 100=\mathrm{V} 1$ indicates a main frequency of $\approx 4.48 \mathrm{c} / \mathrm{d}$ and a semi-amplitude of $0.04 \mathrm{mag}$ in V. Star \#265 = V2 is probably a new $\delta$ Sct star with a main frequency of $\approx 16.77 \mathrm{c} / \mathrm{d}$ and a semi-amplitude of 0.02 mag only in $\mathrm{V}$. The light curves of two individual nights are presented in Fig. 2.

\subsection{NGC 6811}

\subsubsection{Analysis}

The observed field of NGC 6811 is illustrated by Fig. 3. We used the Lindoffnumbering for identification purposes (same as in (WE)BDA). 230 stars received an internal numbering. 441 stars were included in the reduction scheme and 
examined for rapid light variations. The CCD data consist of more than 200 differential magnitudes in the mean for most targets with actual numbers varying between 56 (a few) and 355 (many) depending on the position in the field.

\subsubsection{Discussion of individual variable stars}

We report the detection of nine new variable stars in the field, of which six are probably short-periodic variable stars of type $\delta$ Scuti and one is a suspected short-period variable. The light curves of the variable stars detected in NGC6811 are presented in Fig. 4. Stars \#18=V1 and \#37 = V2 are two $\delta$ Sct type variables, with a main frequency of about $20 \mathrm{c} / \mathrm{d}$ and a semi-amplitude of the order of $0.01 \mathrm{mag}$ in $\mathrm{V}$. Star $\# 70=\mathrm{V} 3$ is a $\delta$ Sct type variable with a main frequency of about $7.5 \mathrm{c} / \mathrm{d}$ and a semi-amplitude of $0.02 \mathrm{mag}$ in $\mathrm{V}$. The data of star \#39 = V4 indicate a frequency of about $8.4 \mathrm{c} / \mathrm{d}$ with a semi-amplitude of $0.01-0.02 \mathrm{mag}$ in $\mathrm{V}$. Star \#113 = V5 is a suspected variable of a similar type with a possible frequency of $13 \mathrm{c} / \mathrm{d}$ of much smaller amplitude $(<0.01 \mathrm{mag})$. Two $\delta$ Scuti variable stars were also detected among the new designations: these are stars \#489 = V6 and \#491 = V7 (following the BDA-numbering) with frequencies of $\approx 9.4 \mathrm{c} / \mathrm{d}$ and possibly $15 \mathrm{c} / \mathrm{d}$ and semi-amplitudes of 0.04 and $0.02 \mathrm{mag}$ in $\mathrm{V}$ respectively. Two more variable stars of unknown type ( $\mathrm{V} 8$ and V9) were discovered at the field edges (Fig. 5, left): they were only observed very shortly (for about $3 \mathrm{hrs}$ ) and do not show the characteristics typical of $\delta$ Scuti stars as their periods are longer. The right panel of Fig. 5 shows the observed light variations with a large amplitude in both cases.

\subsection{NGC 7209}

\subsubsection{Analysis}

The observed field of NGC 7209 is shown in Fig. 6. We used the Mäversnumbering for identification purposes (same as in (WE)BDA). 215 stars received an internal numbering. 357 stars were included in the reduction scheme and examined for rapid light variations. The CCD data consist of more than 900 differential magnitudes for most targets with actual numbers varying between 70 (a few) and 1100 (many) depending on the position in the field.

\subsubsection{Discussion of individual variable stars}

Although we did not (yet) systematically inspect all the material for this cluster, we already report the detection of three possible $\delta$ Scuti variable stars in the field: stars \#24 = V1 (= GSC 3605 2253), \#55 = V2 (= GSC 3605 2309) and \#59 = V3 (= GSC 36052247 1). Stars \#24 and \#55 are probably $\delta$ Scuti 
stars with rather small semi-amplitudes $(<0.01 \mathrm{mag})$. The data of star \#59 clearly indicate a frequency of $7.7 \mathrm{c} / \mathrm{d}$ with a semi-amplitude of $0.01 \mathrm{mag}$ in V. The observed light variations of the detected variabe stars in NGC7209 are plotted in Fig. 7. In addition, star \#112 = V4 (= GSC $36052721=$ SAO 51648), an infra-red source, appears to show small but irregular fluctuations.

We also noted the existence of slower fluctuations with observed amplitudes of the order of 0.1-0.2 mag in the $V$ magnitudes of a few stars which were however only occasionally observed: \#91 (2 nights showing a (start of a) minimum compared to the rest of the data), \#122 (2 nights, $\Delta V=0.3 \mathrm{mag}), \# 179$ (1 night, $\Delta \mathrm{V}=0.6 \mathrm{mag}$ ) and \#3612 (1 night, $\Delta \mathrm{V}=0.6 \mathrm{mag}$ ) (using the BDA-numbering).

\section{Figures for three open clusters}

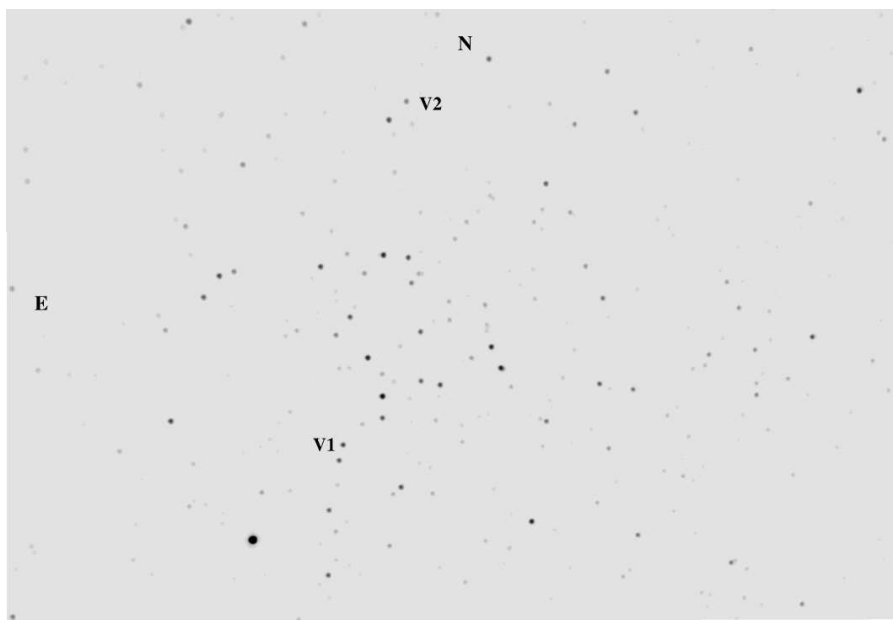

Figure 1: Sample CCD frame of the open cluster NGC 1664. The newly detected variable stars are indicated. 

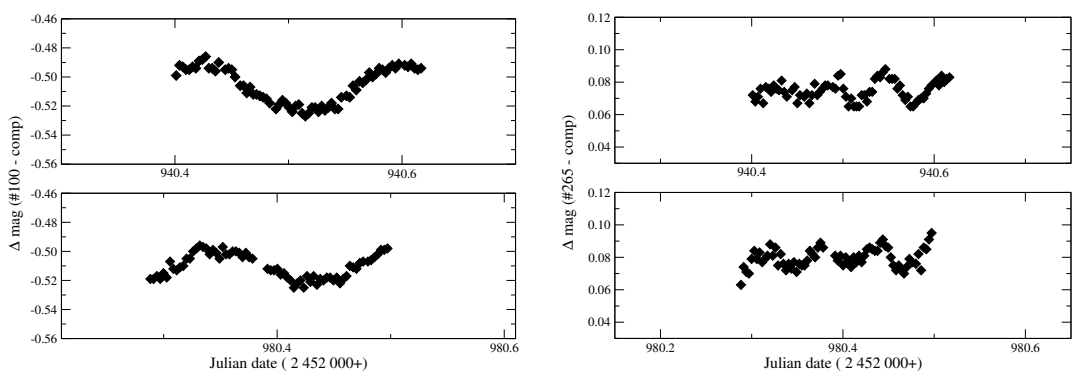

Figure 2: Light curves of NGC1664. Left panels: star \#100. Right panels: star \#265.

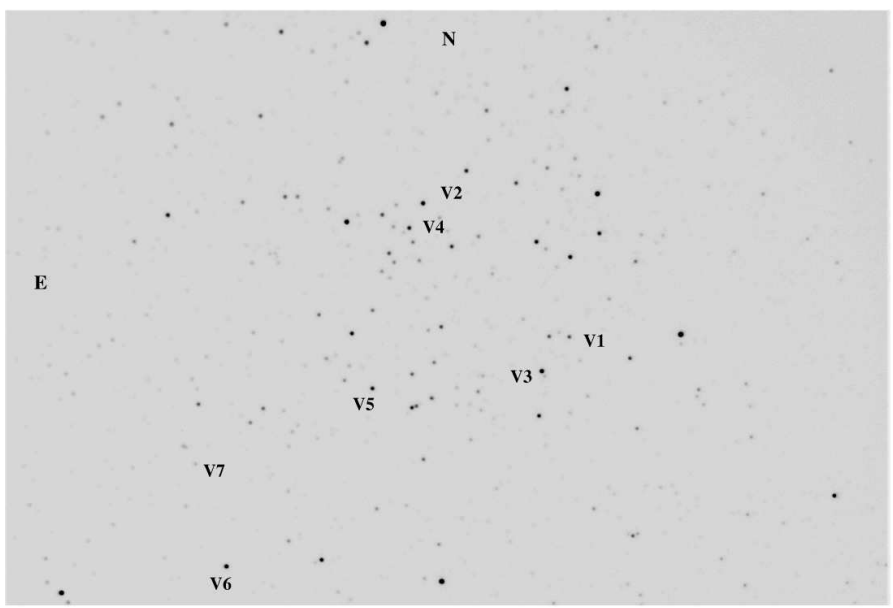

Figure 3: Sample CCD frame of the open cluster NGC 6811. The newly detected variable stars are indicated 

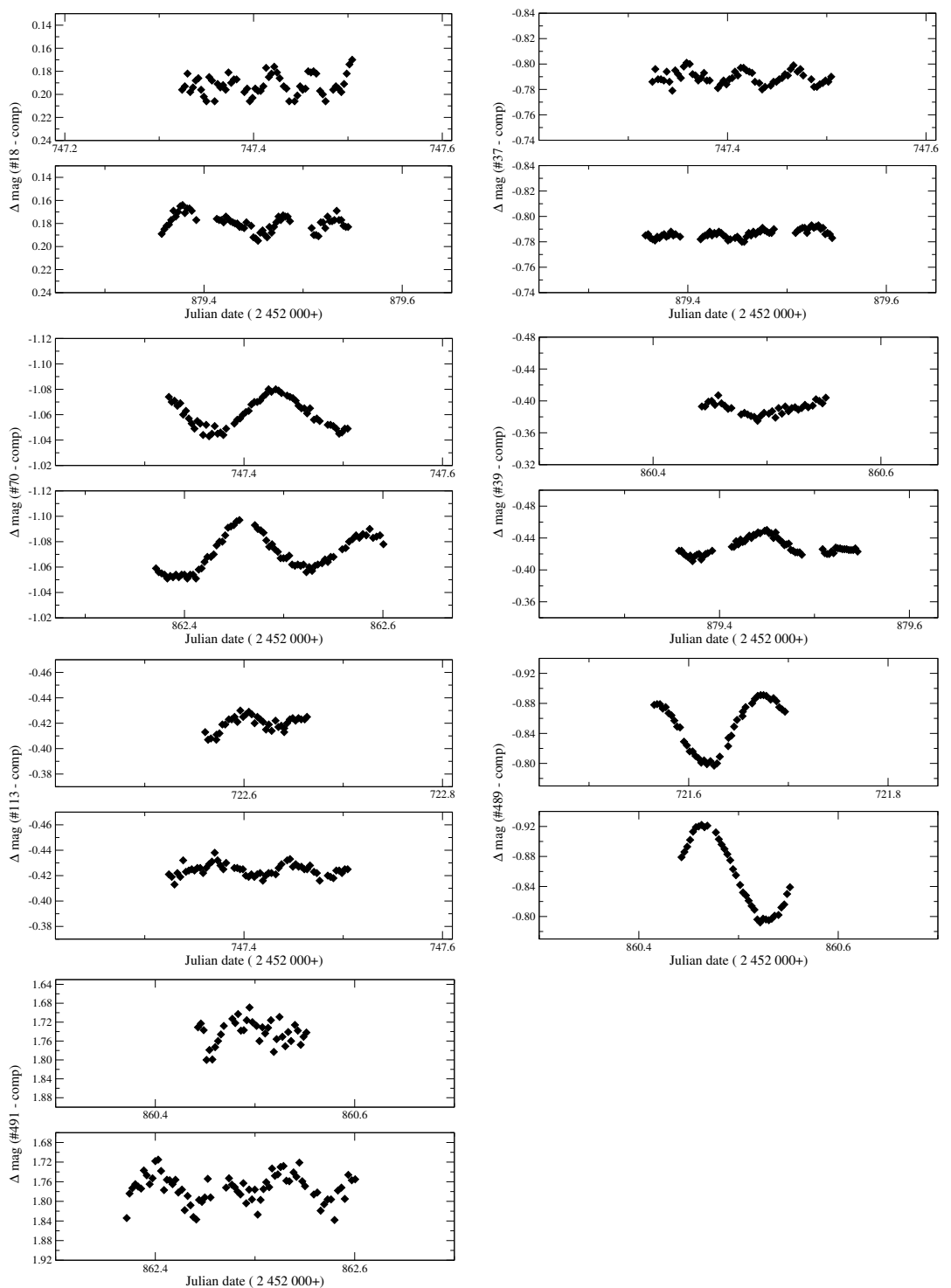

Figure 4: Light curves of NGC6811. From top to bottom and left to right: stars \#18, \#37, \#70, \#39, \#113, \#489, \#491. 

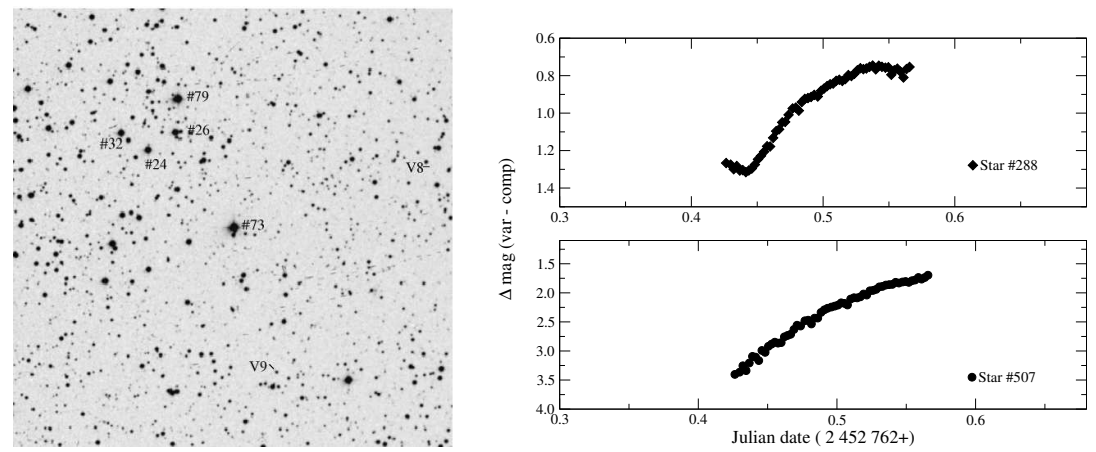

Figure 5: Left panel: Extended field of the open cluster NGC 6811 (source: Digitized Sky Survey). The newly detected variable stars are indicated. Right panels: Light curves of NGC6811,V8-V9.

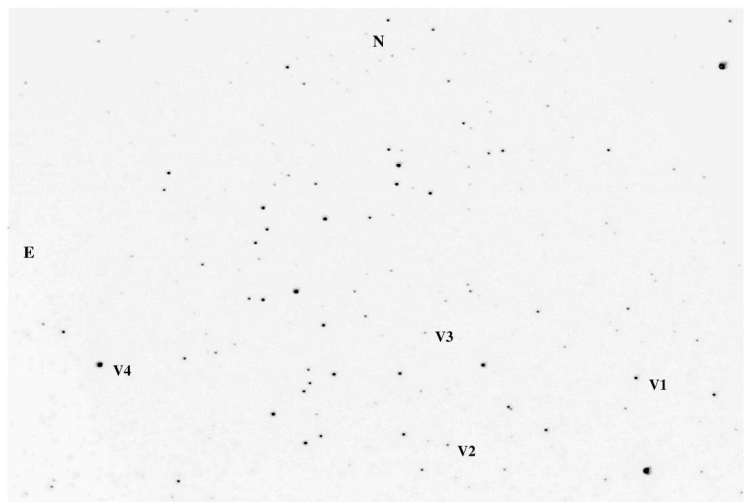

Figure 6: Sample CCD frame of the open cluster NGC 7209. The newly detected variable stars are indicated. 

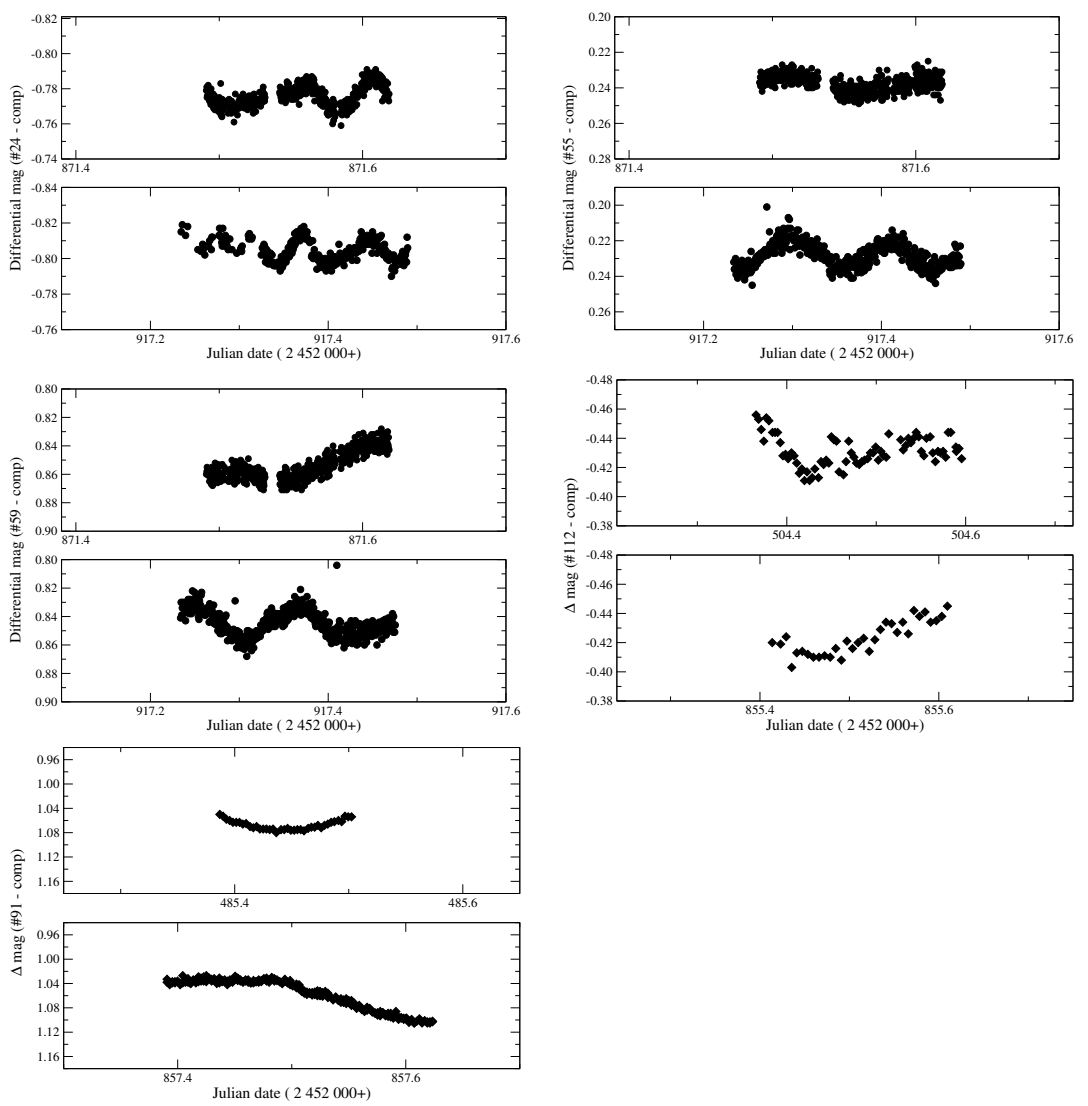

Figure 7: Light curves of NGC7209. From top to bottom and left to right: stars \#24, \#55, \#59, \#112, \#91. 


\section{Conclusions}

We gathered new time-series CCD observations in two filters with the aim to search for short-periodic variable stars in three poorly studied open clusters from the STACC list: NGC 1664, NGC 6811 and NGC 7209. The time series data are particularly extensive in the case of NGC 7209 (more than 100 hrs of observations). Among our first results we report the detection of two possible $\delta$ Scuti variable stars in the field centered on NGC 1664, the detection of nine new variable stars in the field centered on NGC 6811 of which six are probably of type $\delta$ Scuti and one is a suspected $\delta$ Scuti star as well as the detection of three probable $\delta$ Scuti variable stars in the field centered on NGC 7209. The search in NGC 7209 will be resumed as we did not (yet) systematically inspect all the material available. It is also our intention to publish the BV-photometry and to provide updated color-magnitude diagrams for these open clusters.

Acknowledgments. This work is based on CCD observations collected at Beersel Hills (BHO $40 \mathrm{~cm}, \mathrm{BHO} 25 \mathrm{~cm}$ ), Hoher List (1m Cass.), NAO Rozhen (Schmidt 50/70cm), SETEC (30cm Meade LX-200) and Skinakas (1.3m) observatories. The Skinakas Observatory is a collaborative project of the University of Crete, the Foundation for Research and Technology - Hellas, and the MaxPlanck-Institut für Extraterrestrische Physik. We warmly thank Dr. P. Wils for assistance with the reductions. We are grateful to the respective directors Dr. K. Reif, Prof. K. Panov and Prof. I. Papamastorakis for the allocated telescope time. Part of these data were acquired with equipment purchased thanks to a research fund financed by the Belgian National Lottery (1999). $\mathrm{PL}$ and $\mathrm{AS}$ acknowledge support from the Belgian Science Policy and from the Bulgarian Academy of Sciences (project BL/33/B11). PVC is grateful to the Royal Observatory of Belgium for putting at his disposal material acquired by the Fund for Scientific Research - Flanders (Belgium) (project G.0178.02). The Simbad (Centre de Données Astronomiques, Strasbourg, France) and the (WE)BDA (Institut d'Astronomie, Lausanne, Switserland) databases were extensively used.

\section{References}

Baumgardt, H., Dettbarn, C. \& Wielen, R. 2000, A\&AS 146, 251

Bessell, M.S. 1995, CCD Astronomy 2, No. 4, 20

Dias, W.S., Lepine, J.R.D. \& Alessi, B.S. 2001, A\&A 376, 441

Frandsen, S. \& Arentoft, T. 1998, The Journal of Astronomical Data, Vol. 4, No. 6 Freyhammer, L. M., Arentoft, A. \& Sterken, C. 2001, A\&A 368, 580

Glushkova, E.V., Batyrshinova, V.M. \& Ibragimov, M. A. 1999, Astron. Lett. 25, 86 Honeycutt, R.K. 1992, PASP, 104, 435

Kjeldsen, H. \& Frandsen, S. 1992, PASP 104, 413 
Mermilliod J.-C. 1995, in "Information and On-Line Data in Astronomy",

Eds D. Egret \& M.A. Albrecht (Kluwer Academic Press, Dordrecht), 127

Missana, M. \& Missana, N. 1998, Astronomische Nachrichten 319, 187

Peña, J. \& Peniche, R. 1994, Rev. Mex. de Astronomía y Astrofísica 28, 139

Platais, I. 1991, A\&AS 87, 557

Sanders, W.L. 1971, A\&A 15, 368

Tsvetkov, M.K., Georgiev, T. B., Bilkina, B.P., Tsvetkova, A. G. \& Semkov, E.H. 1987, Bolgarskaia Akademiia Nauk, Doklady, Vol. 40, No. 5, 9

Vancevicius, V., Platais, I., Paupers, O. \& Abolins, E. 1997, MNRAS 285, 871 